\title{
ON THE CESÀRO SUMMABILITY OF ULTRASPHERICAL SERIES
}

\author{
ALOK VERMA AND ARAOHANA SHARMA
}

\begin{abstract}
In this paper the Cesàro summability of the ultraspherical series has been investigated which extend and generalize the results of Wang $[5,6]$ of Fourier series.
\end{abstract}

1. Let $f(\theta, \phi)$ be a function defined for the range $0 \leq \theta \leq \pi, 0 \leq \phi \leq 2 \pi$ on a sphere $S$. The ultraspherical series associated with this function is,

$$
f(\theta, \phi) \sim \frac{1}{2 \pi} \sum_{n=0}^{\infty}(n+\lambda) \iint_{S} \frac{P_{n}^{(\lambda)}(\cos w) f\left(\theta^{\prime}, \phi^{\prime}\right) d \sigma^{\prime}}{\left[\sin ^{2} \theta^{\prime} \sin ^{2}\left(\phi-\phi^{\prime}\right]^{1 / 2-\lambda}\right.} ; \quad \lambda>0
$$

where $w$ is the spherical distance between the points $\left(\theta^{\prime}, \phi^{\prime}\right)$, i.e.

$$
\cos w=\cos \theta \cos \theta^{\prime}+\sin \theta \sin \theta^{\prime} \cos \left(\phi-\phi^{\prime}\right)
$$

and $d \sigma^{\prime}=\sin \theta^{\prime} d \theta^{\prime} d \phi^{\prime}$

The Laplace series is a particular case of the series of (1.1) for $\lambda=1 / 2$, while it reduces to the trigonometric series in the limit as $\lambda \rightarrow 0$, because

$$
\lambda \stackrel{\lim }{\longrightarrow} 0 \quad \frac{1}{\lambda} P_{n}^{(\lambda)}(\cos \theta)=\frac{2}{n} \cos n \theta, \quad n \geq 1 .
$$

The ultraspherical polynomials $P_{n}^{(\lambda)}(x)$ are defined by the following expansion:

$$
\left[1-2 x z+z^{2}\right]^{-\lambda}=\sum_{n=0}^{\infty} z^{n} P_{n}^{(\lambda)}(x), \quad \lambda>0 .
$$

We suppose throughout that the function

$$
f\left(\theta^{\prime}, \phi^{\prime}\right)\left[\sin ^{2} \theta^{\prime} \sin ^{2}\left(\phi-\phi^{\prime}\right)\right]^{\lambda-1 / 2}
$$

Received May 13, 1994; revised September 26, 1994. 
is integrable $(L)$ over the whole surface of the unit sphere and following Kogbetliantz [2] we define the generalised mean value of $f(\theta, \phi)$ as follows:

$$
f(w)=\frac{1}{2 \pi(\sin w)^{2}} \int_{C_{v}} \frac{f\left(\theta^{\prime}, \phi^{\prime}\right) d S^{\prime}}{\left[\sin ^{2} \theta^{\prime} \sin ^{2}\left(\phi-\phi^{\prime}\right)\right]^{1 / 2-\lambda}}
$$

where the integral is taken along the small circle $C_{w}$, where $d s^{\prime}$ is element of the arc of $C_{w}$, whose centre is $(\theta, \phi)$ on the sphere and whose curvilinear radius is $w$.

We write,

and

$$
\begin{aligned}
\phi(w) & =\left[f(w)-\frac{A \Gamma(\lambda)}{\Gamma(1 / 2) \Gamma(1 / 2+\lambda)}\right](\sin w)^{2 \lambda} \\
\Phi_{p}(x) & =\frac{1}{\Gamma(p)} \int_{0}^{x}(x-t)^{p-1} \phi(t) d t \\
\Phi_{0}(x) & =\phi(x) \\
\phi_{p}(x) & =\Gamma(p+1) x^{-p} \Phi_{p}(x), \quad p \geq 0
\end{aligned}
$$

$$
\Phi_{p}(x)=\frac{d}{d x} \Phi_{p+1}(x), \quad-1<p<0
$$

The authors have obtained a theorem for Cesàro summability of the series (1.1) analogous to those of Izumi and Sonouchi [1]. The object of this paper is to extend and generalize the result of Wang [5 \& 6] foFr the same series.

We prove the following:

Theorem. If $y>x>0$ and

$$
\Phi_{x}(t)=o\left(t^{y+2 \lambda+1 / \lambda}\right) \quad \text { for } o<\lambda<1 \text {. }
$$

then the series (1.1) is summable $(C, \alpha+\lambda)$ at the point $(\theta, \phi)$, where

$$
\alpha=\frac{m y+x-y}{m+y-x}
$$

and $m$ is a positive integer, such that $m \geq x>m-1$.

2. For the proof of the theorem we require the following Lemmas:

Lemma 1. Let $S_{n}^{k}(w)$ denote the $n^{\text {th }}$ Cesàro mean of order $k$ of the series

$$
\sum(n+\lambda) P_{n}^{(\lambda)}(\cos w)
$$


Then we have, for $\lambda>0$ and $p \geq 0$,

$$
S_{n}^{p}(w)=\frac{d^{p}\left(S_{n}^{k}(w)\right)}{d w^{p}}=\left\{\begin{array}{ll}
O\left(n^{2 \lambda+p+1}\right) & \text { for } 0 \leq w \leq \pi, k>0 \\
O\left(\frac{n^{\lambda+p-k}}{w^{\lambda+k+1}}\right)+O\left(\frac{1}{n w^{2 \lambda+p+2}}\right) & \text { for } 0<w \leq a<\pi \\
O\left(\frac{n^{\lambda+p-k}}{w^{k+\lambda+1}}\right) & \text { for } 0<w \leq a<\pi \\
\lambda+1+[p] \geq k
\end{array}\right. \text { and }
$$

Lemma 2. In order that the series (1.1) be summable $(C, k)$, it is sufficient that the integral

$$
i=\int_{0}^{\delta} \phi(w) S_{n}^{k}(w) d w=o(1)
$$

For $0<\delta<\pi$ and for each $k>\lambda$.

Lemma 3. For a non-integral

$$
\delta=m+\sigma, \quad(0<\sigma<1)
$$

We have

$$
\int_{0}^{\Delta} \Phi_{\delta}(u) S_{n}^{(\delta)}(u) d u=\Phi_{m+1}(\Delta) S_{n}^{(m)}(\Delta) \times \int_{0}^{\Delta} \Phi_{m}(t) S_{n}^{(m)}(t) d t
$$

Lemma 4. If $0 \leq u \leq \frac{1}{n}$

$$
F(n, u)=O\left(n^{2 \lambda+m+1} u^{m-x}\right)+O\left(n^{2 \lambda+m+1} u^{m-x-1}\right)
$$

where

$$
F(n, u)=\Gamma \frac{1}{(m-x)} \int_{u}^{1 / n}(t-u)^{m-x-1} S_{n}^{(m)}(t) d t
$$

Lemmas 1, 2, 3 and 4 are due to Obrechkoff [3] and Singhai [4] respectively.

\section{Proof of the Theorem}

In order to prove the theorem, in view of (2.3) it is sufficient to show that

$$
n \stackrel{\lim }{\longrightarrow} \infty \int_{0}^{\delta} \phi(u) S_{n}^{\alpha+\lambda}(u) d u=0
$$

under the condition of the theorem. 
We have the following inequality:

$$
x>\alpha>m-1 \quad \text { (F. T. Wang) }
$$

Also we have

$$
x+1>m \geq x
$$

Then

$$
\begin{aligned}
i & =\int_{0}^{\delta} \phi(w) S_{n}^{k}(w) d w \\
& =\left[\sum_{p=1}^{m}(-1)^{p-1} \Phi_{p}(w)\left(\frac{d}{d w}\right)^{p-1} S_{n}^{\alpha+\lambda}(w)\right]_{0}^{\delta}+(-1)^{m} \int_{0}^{\delta} \Phi_{m}(t) S_{n}^{(m)}(t) d t . \\
& =I_{1}+(-1)^{m} I_{2}
\end{aligned}
$$

Since $\alpha>m-1$

$$
I_{1}=o(1) \quad \text { as } \quad n \longrightarrow \infty
$$

We write

$$
\begin{gathered}
I_{2}=\int_{0}^{\delta} \Phi_{m}(t) S_{n}^{(m)}(t) d t \\
=\int_{0}^{1 / n}+\int_{1 / n}^{\delta}=I_{2.1}+I_{2.2} \\
I_{2.2}=\left[\Phi_{m}(t) S_{n}^{(m-1)}(t)\right]_{1 / n}^{\delta}-\int_{1 / n}^{\delta} \Phi_{m-1}(t) S_{n}^{(m-1)}(t) d t \\
=I_{2.2 .1}-I_{2.2 .2}
\end{gathered}
$$

we have

Also if we write

$$
\begin{aligned}
I_{2.2 .1} & =o(1)+o\left(\frac{1}{n^{y+2 \lambda+1 / \lambda}}\right) O\left(\frac{n^{m-1-\alpha}}{n^{-\alpha-1-2 \lambda}}\right) \\
& =o(1) \text { as } n \longrightarrow \infty
\end{aligned}
$$

then

$$
\Phi^{*}(t)=\int_{0}^{t}\left|\Phi_{m-1}(u)\right| d u
$$

$$
\begin{aligned}
I_{2.2 .2}= & O\left[n^{m-1-\alpha} \int_{1 / n}^{\delta} \frac{\left|\Phi_{m-1}(t)\right|}{t^{\alpha+2 \lambda+1}} d t\right] \\
= & O\left(n^{m-1-\alpha}\right)\left[\frac{\Phi^{*}(t)}{t^{\alpha+2 \lambda+1}}\right]_{1 / n}^{\delta}+O\left(n^{m-1-\alpha}\right) \int_{\frac{1}{n}}^{\delta} \frac{\Phi^{*}(t)}{t^{\alpha+2 \lambda+2}} d t \\
= & O\left(n^{m-1-\alpha}\right)+O\left(n^{m-1-\alpha}\right) \circ\left(\frac{1}{n^{y+2 \lambda+1 / \lambda}}\right) \\
& \times n^{\alpha+2 \lambda+1}+O\left(n^{m-1-\alpha}\right) \int_{1 / n}^{\delta} \frac{o\left(t^{y+2 \lambda+1 / \lambda}\right)}{t^{\alpha+2 \lambda+2}} d t \\
= & O(1) \text { as } n \longrightarrow \infty
\end{aligned}
$$


When $x$ is not an integer.

$$
\begin{aligned}
I_{2.1} & =\int_{0}^{1 / n} \Phi_{m}(t) S_{n}^{(m)}(t) d t \\
& =\int_{0}^{1 / n} \Phi_{x}(u) F(n, u) d u
\end{aligned}
$$

where $F(n, u)=\frac{1}{\Gamma(m-x)} \int_{u}^{1 / n}(t-u)^{m-x-1} S_{n}^{(m)}(t) d t$

Now

$$
\begin{aligned}
I_{2.1}= & o\left[\int_{0}^{1 / n}(y+2 \lambda+1 / \lambda) O\left(n^{2 \lambda+m+1} u^{m-x}\right) d u\right] \\
& +o\left[\int_{0}^{1 / n} u^{(y+2 \lambda+1 / \lambda)} O\left(n^{2 \lambda+m+1} u^{m-x-1}\right) d u\right] \\
= & o(1) \text { as } n \longrightarrow \infty
\end{aligned}
$$

When $x=m$ is an integer.

$$
\begin{aligned}
I_{2.1} & =\int_{0}^{1 / n} \Phi_{x}(t) S_{n}^{(x)}(t) d t \\
& =o\left[\int_{0}^{1 / n} u^{y+2 \lambda+1 / \lambda} O\left(n^{2 \lambda+x+1}\right) d u\right] \\
& =o(1) \text { as } n \longrightarrow \infty
\end{aligned}
$$

combining (3.1), (3.2), (3.3), (3.4) and (3.5) the result is proved.

\section{Acknowledgement}

The authors would like to acknowledge his indebtedness to Dr. G.S.Pandey, Professor and Head, School of Studies in Mathematics, Vikram University, Ujjain (M.P.) India for his valuable guidence during the preparation of this paper. We also express our gratitude to the referee for his valuable suggestions which brings the paper to the present form.

\section{References}

[1] S. Izumi and G. Sonouchi, "Notes on Fourier Anaysis (XXXIX)," Theorems concerning Cesàro Summability Tohoku Math. Jour., Vol. 1.2 Second Series, 313-326, 1949-51.

[2] E. Kogbetliantz, "Researches sur la sommabilite des series ultraspherique par la methode des moyennes arithmetiques," Jour. Math. (9)3, 107-187, 1924.

[3] N. Obrechkoffe, "Sur la sommation de la, series ultraspherique par la, methode des moyennes arthmetiques," Rend. del. cir. Mate. di Palermo, 59, 266-237, 1932.

[4] B. C. Singhai, Cesaro summability of ultraspherical series, Annali di Matematica pura ed applicata (1961). 
[5] F. T. Wang, "A. note on Cesaro Summability of Fourier series," Ann. Math., 44, 397-400, 1943.

[6] F. T. Wang, "A remark on (c) summability of Fourier Series," Jour. London, Math. Soc. 22, 40-47, 1947.

Department of Mathematics, Govt. Science P.G. College, Bilaspur (M.P.), India 495001. 